\title{
Prevalence of Symptoms of Obstructive Sleep Apnoea in Children Undergoing Routine Adenotonsillectomy
}

\author{
Swagata Khanna, Sunil KC, Mahamaya Prasad Singh \\ Department of ENT, Gauhati Medical College \& Hospital, Guwahati, India \\ Email: swagatakhanna@sify.com
}

Received August 1, 2012; revised September 12, 2012; accepted October 1, 2012

\begin{abstract}
Introduction: Obstructive sleep apnoea (OSA) is a condition characterized by episodic partial or complete obstruction of the upper airway during sleep leading to apnoea or cessation of breathing. Obstruction of the upper airway during sleep may result in the generation of noise (snoring), reduction (hypopnoea) or cessation (apnoea) of airflow at the nostrils and mouth. There are multiple indications for undertaking a patient for adenoidectomy and/or tonsillectomy with obstructive sleep apnoea (OSA) being one among many. Objective: The aim of the present study was to find the prevalence of OSA symptoms in children undergoing adenotonsillectomy for indications other than that of obstructive sleep apnoea. Material \& Methods: The study was conducted in the Department of ENT and Head \& Neck surgery, Gauhati Medical College \& Hospital, Guwahati for a period of one year. Twenty six patients who underwent adenoidectomy and/or tonsillectomy during this period were selected for the study. The parents of the patients were administered the Paediatric Sleep Questionnaire pre-operatively and the patients were evaluated for any symptoms of OSA. A score of 8 or more was suggestive of presence of breathing related sleep disorder. All statistical analyses were performed using statistical software SPSS 16.0 version. To test for the difference in the proportion between different variables, chisquare/fisher exact test where appropriate were employed. All statistical tests were two tailed with 0.05 as the threshold level of significance. Results: 11 children (42.3\%) had a score of 8 or more out of the 26 children in Paediatric sleep questionnaire. The chi square for this was 4.696 with a p value of 0.096 . The snoring subscale was found to be positive in 19 children $(73.1 \%)$. All children with score of 8 or more were positive for the snoring scale. The sleepiness subscale was found to be positive in 14 children (53.8\%). 10 of the 11 children were positive for sleepiness scale among the children who had a score of 8 or more in the questionnaire. These were found to be statistically significant. Conclusion: A significant population of the children undergoing routine adenotonsillectomy also has symptoms of obstructive sleep apnoea. The pathophysiology of obstructive sleep apnoea should be borne in mind in all children having adenotonsillar hypertrophy and a prompt and early intervention into these children should be aimed for both the infective etiology and the possible outcomes of their compromise to the airway column for a better quality of life.
\end{abstract}

Keywords: Adenotonsillectomy; Obstructive Sleep Apnoea

\section{Introduction}

The nasopharyngeal lymphoid aggregate or Lushka's tonsil was coined the term Adenoid by Wilhelm Meyer. The adenoid forms part of Waldeyer's ring of lymphoid tissue at the portal of the upper respiratory tract. In early childhood this is the first site of immunological contact for inhaled antigens. Palatine tonsils consist of paired aggregates of lymphoid tissue. They are located in the pocket formed between the palatoglossus and palatopharyngeus muscles and the overlying folds of mucosa, which make up the anterior and posterior tonsillar pillars. This forms the first site of immunological contact for ingested antigens.

Obstruction of the upper airway during sleep may result in the generation of noise (snoring), reduction (hy- popnoea) or cessation (apnoea) of airflow at the nostrils and mouth. Obstructive sleep apnoea (OSA) is a condition characterized by episodic partial or complete obstruction of the upper airway during sleep which occurs as a consequence of an anatomical reduction in the upper airway or in-coordination of upper airway dilatory muscle activity. Most commonly, it may be due to the combination of both these factors [1-5]. Intermittent episodes of brief cessation of breathing may be physiological. OSA is defined as a cessation of airflow for more than 10 seconds despite continuing ventilatory effort, 5 or more times per hour of sleep and a decrease of more than $4 \%$ in $\mathrm{S}_{\mathrm{a}} \mathrm{O}_{2}$. Six seconds or less may be pathological in children. The most common cause of OSA in children is hypertrophy of adenoid and palatine tonsils and adenotonsillectomy is the curative procedure in most cases [6-9]. 
There are multiple indications for undergoing adenoidectomy and/or tonsillectomy. The objectives of the present study were to find the prevalence of OSA symptoms in children undergoing adenotonsillectomy for indications other than that of obstructive sleep apnoea and to find the association of symptoms of OSA, Snoring scale and sleepiness scale with type of surgery among the children who underwent routine adenotonsillectomy. There is no association between the symptoms of OSA, Snoring scale and Sleepiness scale with type of surgery among the children who underwent routine adenotonsillectomy.

\section{Materials \& Methods}

The study was conducted in the Department of ENT and Head \& Neck surgery, Gauhati Medical College \& Hospital, Guwahati; from 1st August 2010 to 31st July 2011.

26 patients (16 male and 10 female) in the age group of 5 to 15 years who underwent adenoidectomy and/or tonsillectomy during this period were selected for the study. The cases selected for the study were subjected to detailed history taking and examination, a routine haemogram (HB, ESR, BT, CT, TC, DC) urine examination (albumin, sugar, and microscopy) and stool examination (ova, cyst). All the patients in active stage of the disease were treated with a course of suitable antibiotic, systemic antihistamines and local decongestants. Specific investigations like pure-tone audiometry, tympanometry, $\mathrm{X}$-ray of the nasopharynx was done in indicated patients.

A standardized clinical data sheet consisting of questions regarding child's snoring patterns, night time and day time symptoms, as well as other symptoms associated with OSA. The parents of the patients were administered this Paediatric Sleep Questionnaire [10] pre-operatively and the patients were evaluated for any symptoms of OSA. A score of 8 or more was suggestive of presence of breathing related sleep disorder.

All statistical analyses were performed using statistical software SPSS 16.0 version. Quantitative variables like age, weight were summarized using mean or median and to understand the variation in the data, standard deviation (SD)/interquartile range were calculated wherever appropriate. Qualitative variables such as gender, type of operation underwent, indication for surgery etc were summarized using frequency and percentages. To test for the difference in the proportion between different variables, chi-square/fisher exact test where appropriate were employed. All statistical tests were two tailed with 0.05 as the threshold level of significance.

\section{Results \& Observation}

A total of 26 children were included in the study. 14 (53.8\%) were boys and 12 (46.2\%) were girls with the mean age being 8 years (SD). All the children (26) underwent surgery. Among all the children who underwent surgery, 16 children (61.5\%) underwent Adenoidectomy (Figure 1) 5 (19.2\%) children underwent Tonsillectomy (Figure 2) and 19.2\% underwent Adenotonsillectomy. The most common indication for the surgery was Otitis media with effusion and recurrent sinusitis (34.6\%). Recurrent sore throat was the indication in 7 children (26.9\%) while peri tonsillar abscess was the indication in one child (3.8\%).

Among the 26 children who underwent surgery, 16 children complained of some sort of hearing impairment and they were subjected to pure tone audiometry and impedance audiometry. On pure tone audiometry, 6 children (37.5\%) were found to have bilateral mild conductive hearing loss while bilateral moderate conductive hearing loss was found in 5 children (31.2\%). 3 children (18.8\%) showed unilateral mild conductive hearing loss while in 2 children (12.5\%) unilateral moderate conductive hearing loss was noted. On impedance audiometry, 13 children (81.2\%) showed bilateral ' $\mathrm{B}$ ' type with no

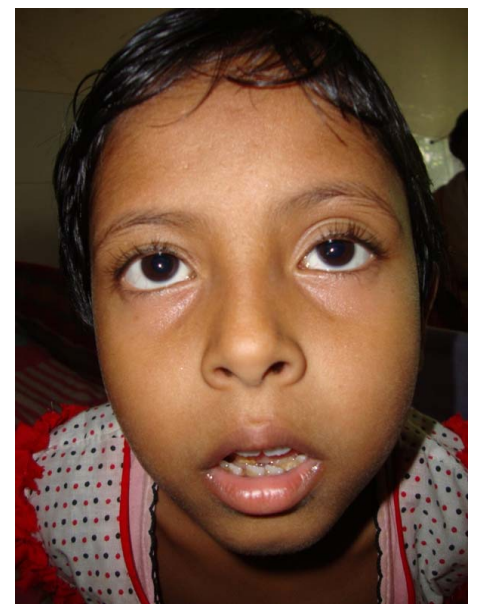

Figure 1. Photograph of a patient with adenoids showing open mouth breathing and expression less face (Adenoid facies).

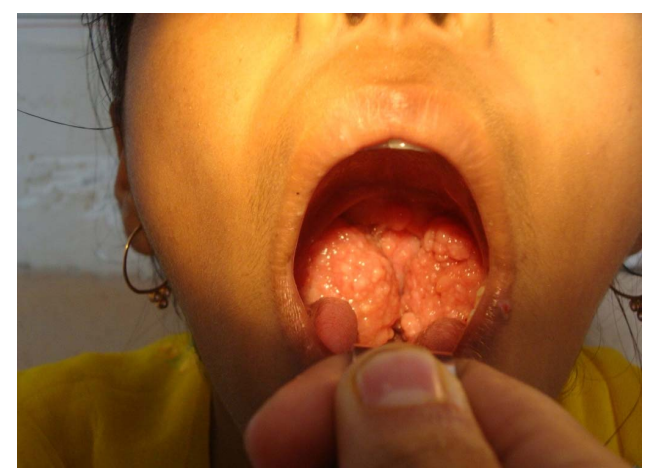

Figure 2. Photograph of a patient showing follicular hypertrophy of bilateral palatine tonsils leading to compromise of the oropharyngeal respiratory pathway. 
reflex while 3 children (18.8\%) showed unilateral "B" type hypertrophied adenoids in 22 children (84.6\%)while it was found to be normal in 4 children (15.4\%) (Figure 3).

As shown in Table 1, 11 children (42.3\%) had a score of 8 or more out of the 26 children. Among the children who underwent adenoidectomy, 8 children (50\%) had a score of 8 or more in Pediatric sleep questionnaire. The chi square for this was 4.69 with a p value of 0.096 . Trouble or struggle to breathe was seen in 23 (88.5\%) out of 26 children. Chi square was 14.24 and with a p value of 0.001 . Only 5 children (19.2\%) were positive for the history of stop in breathing at night. Day time sleepiness observed by their teacher was seen in 6 children (23.1\%). Dry mouth on waking up in the morning was an uncommon complaint with it being present in 3 (11.5\%) children. $50 \%$ of the children had the history if waking up unrefreshed in the morning. Chi square was 6.200 with a $\mathrm{p}$ value of 0.045 . 22 children (84.6\%) had the history of breathing through the mouth through the day while this complaint was seen in all the children who underwent adenoidectomy, 16 children (100\%). Chi square for this was found to be at 19.855 with a statistically significant $p$ value of 0.001 . Of the 26 children, difficulty in hearing on history was present in 9 children (34.6\%), all of whom underwent adenoidectomy operation. 8.60 was the chi square value with p value being 0.014 . Difficulty in organizing tasks and activities was present in 9 (34.6\%) children.

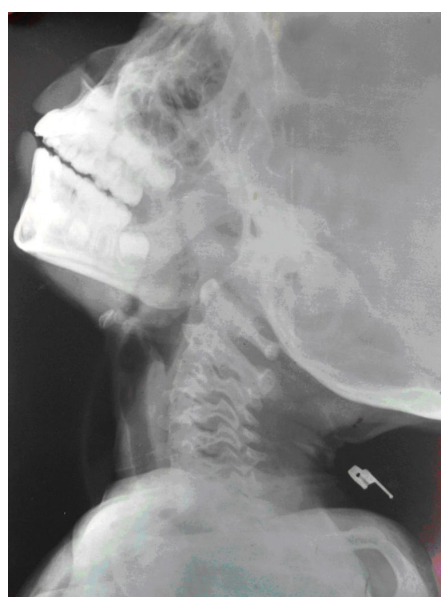

Figure 3. X-ray of the nasopharynx showing hypertrophied nasopharyngeal tonsils compromising the nasal airway.

Table 1. Distribution \& association of symptoms of OSA, snoring scale and Sleepiness scale with type of surgery among the children who underwent routine adenotonsillectomy.

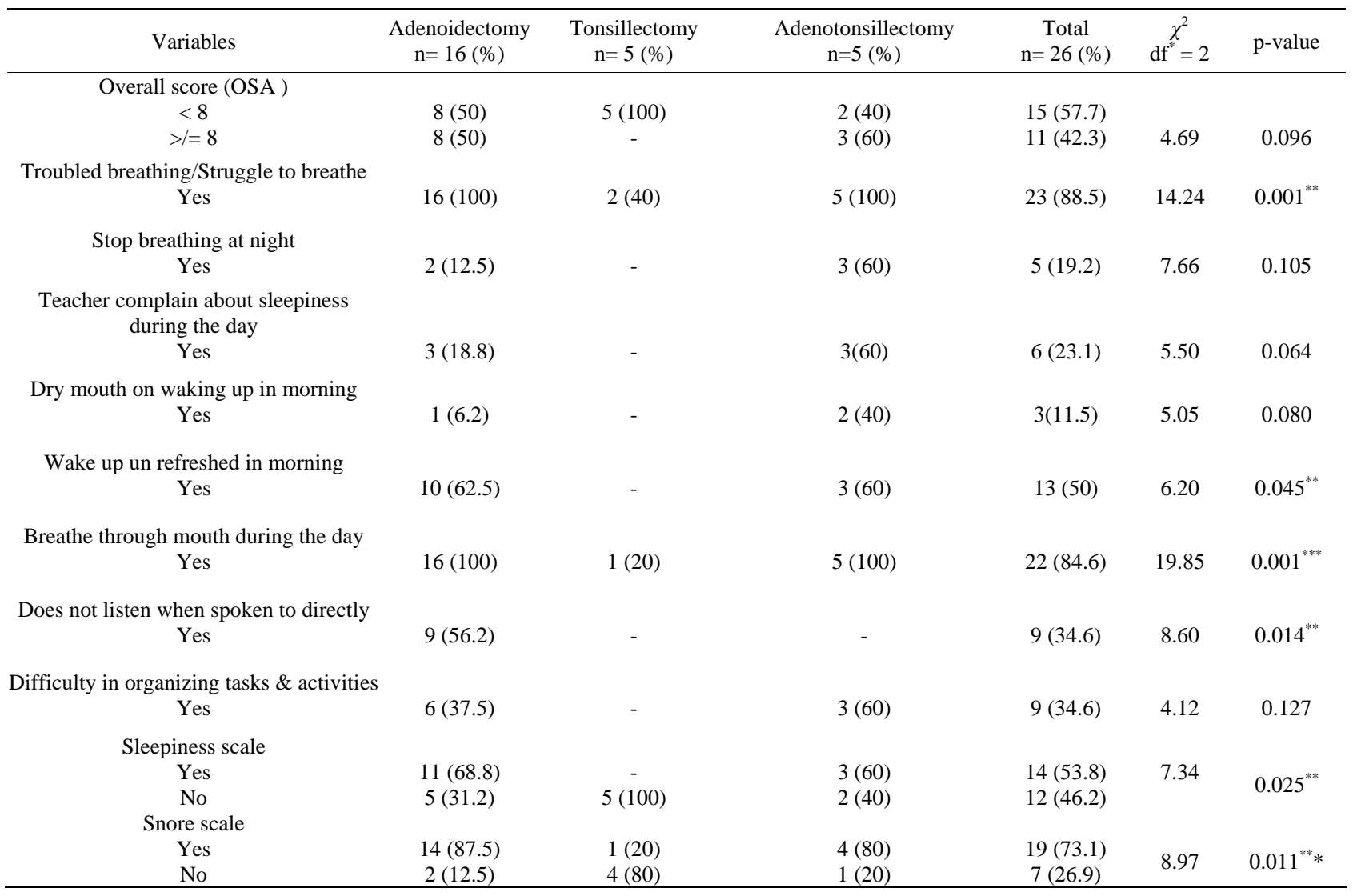

*df: Degree of freedom; ${ }^{* *}$ Statistically significant. 
The snoring subscale includes items A2, A3, A4, and A5 in the questionnaire. The snoring subscale was found to be positive in 19 children (73.1\%), with it being a maximum of $87.5 \%$ in children undergoing adenoidectomy surgery and minimum of $20 \%$ in children undergoing tonsillectomy surgery. All children with score of 8 or more were positive for the snoring scale. These values were found to be statistically significant.

The sleepiness subscale includes items B1, B2, B4, and B6 in the questionnaire. The sleepiness subscale was found to be positive in 14 children (53.8\%), with this being maximum in $68.8 \%$ of children undergoing adenoidectomy operation and was none in children undergoing tonsillectomy surgery. 10 of the 11 children were positive for sleepiness scale among the children who had a score of 8 or more in the questionnaire. This was found to be statistically significant.

\section{Discussion}

Adenoidectomy and/or tonsillectomy are done for a varied number of indications with obstruction to the upper airway passage being one among many. The gold standard diagnostic tool to diagnose OSA is Polysomnography. Polysomnography is resource demanding. Numerous studies have been conducted to find a screening tool to effectively identify patients with clinically significant OSA so as to prioritize Polysomnography and allow prompt treatment. The prevalence of OSA syndrome in children is estimated as $1 \%$ to $3 \%[11,12]$. There have been many studies to correlate clinical findings and polysomnographic findings in patients undergoing adenotonsillectomy for OSA but no studies have been done to identify the co-existence of obstructive sleep apnoea symptoms in children undergoing adenotonsillectomy for other routine indications. Our study aimed at finding this prevalence.

The pediatric sleep questionnaire was used to evaluate the children undergoing adenotonsillectomy. A score of 8 or more in this scale indicated a presence of significant airway obstruction warranting a consultation with a physician [10]. In our study, $42.3 \%$ of the children showed significant amount of airway obstructive symptoms. This was even higher at $50 \%$ in the group of children undergoing adenoidectomy alone while it was $60 \%$ in the children undergoing adenotonsillectomy. None of the children undergoing tonsillectomy were positive for signifycant airway obstruction. Studies have shown that severity of OSA syndrome is not always proportional to the size of the tonsils and adenoids [13]. The pathophysiology of OSA syndrome in children is related to combination of anatomic narrowing and neuromuscular function [14]. Limited space of the nasopharynx compared to that of oropharynx may be the reason for increased symptoms in children undergoing adenoidectomy than the cohort of tonsillectomy children. When both nasopharynx and oropharynx were involved in children undergoing adenotonsillectomy leading to further reduction in space, the symptoms were seen in more number of children.

The peak prevalence of childhood OSA occurs at 2 - 8 years, which is the age when tonsils and adenoids are the largest in relation to the side of collapse [15]. In our study, the mean age of presentation of children with significant symptoms of OSA was 9 years. There was no statistically significant difference in the sex distribution. There were $61.5 \%$ of the children who underwent adenoidectomy as compared to the other group who underwent tonsillectomy alone or adenotonsillectomy. The advent of new and effective antibiotics may be the reason for better control of tonsillitis and its related problem thus leading to a reduced number of children undergoing tonsillectomy. Adenoids on the other hand compromising the narrow nasopharynx and leading to related problems like otitis media with effusion (OME) and recurrent sinusitis warranted a surgery in more number of children. $61.5 \%$ of the children had some form of hearing impairment on objective testing by pure tone audiometry and tympanometry. This might be due to the recurrent acute on chronic inflammation of the adenoid and increased bacterial load which results in squamous metaplasia, reticular epithelium extension, fibrosis of the interfollicular interconnective tissue and reduced mucociliary clearance in children with OME compared to those without OME [16].

On questionnaire evaluation, the complaints of troubled breathing/struggle to breathe, wake up unrefreshed in morning, breathe through mouth during the day, does not listen when spoken to directly were some of the questions which yielded highly significant positives responses in the children. It was also noted that this significant positive response was more in the children who scored 8 or more in the questionnaire evaluation than the rest of the children. Mouth breathing was a complaint seen in $84.6 \%$ of the children and in all the children undergoing adenoidectomy. The findings were the same when X-ray nasopharynx of the children was analyzed; suggesting that the hypertrophied adenoid were the main reason for causation of mouth breathing.

Habitual snoring is a prominent feature of sleep disordered breathing and both are associated with inattentive and hyperactive behavior in children [17-20]. In our study, $73.1 \%$ of the children who underwent surgeries were positive for the snoring subscale and this was found to be statistically significant. All the children who scored 8 or more in the questionnaire were positive for the snoring subscale and were significant statistically. In view of high score on questionnaire along with snoring, these children might be in future exposed to behavioral 
abnormalities that might affect the lifestyle and quality of life of these children.

Excessive daytime sleepiness (EDS) is considered as a key feature of OSA in adults, but its significance remains unclear whether it also occurs frequently in children with OSA. EDS may lead to behavioral problem and impairment of learning skill of the children. The American Academy of Pediatricians lists behavioral problems as symptoms and complications of childhood [21]. In our study, 53.8\% of the children were positive for the sleepiness subscale. 10 of the 11 children were positive for sleepiness scale among the children who had a score of 8 or more in the questionnaire. This was found to be statistically significant.

There are certain limitations of the study. Firstly, a larger cohort of subjects is required to arrive at a conclusion between co-existence of symptoms of OSA and the other indicators for adenotonsillectomy. Lack of a clear guideline to diagnose OSA by clinical methods hinders in the assessment of patients in centers where there is unavailability of polysomnogram. The paucity of literature regarding the prevalence of symptoms of OSA in children undergoing adenotonsillectomy for routine indications hampers our comparison with others studies.

\section{Conclusion}

OSA is a condition that impairs the development and behaviour of growing children. While doing Adenotonsillectomy the condition of OSA should always be kept in mind and should be looked for. Any child undergoing adenotonsillectomy showing features suggestive of OSA should be identified at the earliest and appropriate management given. Despite the limitations, the present study has shown that a significant population of the children undergoing routine adenotonsillectomy also has symptoms of obstructive sleep apnoea. The pathophysiology of obstructive sleep apnoea should be borne in mind in all children having hypertrophy of adenoid and palatine tonsil and a prompt and early intervention into these children should be aimed for both the infective etiology and the possible outcomes of their compromise to the airway column for a better quality of life.

\section{REFERENCES}

[1] M. A. Richardson, A. B. Seid, R. T. Cotton, C. Benton and M. Kramer, "Evaluation of Tonsils and Adenoids in Sleep Apnoea Syndrome,” Laryngoscope, Vol. 90, No. 7, 1980, pp. 1106-1110. doi:10.1288/00005537-198007000-00005

[2] A. E. Sher, "Obstructive Sleep Apnoea Syndrome: A Complex Disorder of the Upper Airway," Otolaryngologic Clinics of North America, Vol. 23, No. 4, 1990, pp. 593-608.
[3] S. T. Kuna and G. Sant’ Ambrogio, "Pathophysiology of Upper Airway Closure during Sleep," Journal of the American Medical Association, Vol. 266, No. 10, 1991, pp. 1384-1389. doi:10.1001/jama.1991.03470100076036

[4] D. W. Roloff and M. S. Aldrich, "Sleep Disorders and Airway Obstruction in Newborns and Infants," Otolaryngologic Clinics of North America, Vol. 23, No. 4, 1990, pp. 639-650.

[5] C. D. Hanning, “Obstructive Sleep Apnoea," British Journal of Anesthesia, Vol. 63, 1989, pp. 648-650. doi:10.1093/bja/63.4.477

[6] J. S. Suen, J. E. Arnold and L. J. Brooks, “Adenotonsillectomy for Treatment of Obstructive Sleep Apnea in Children," Archives of Otolaryngology-Head \& Neck Surgery, Vol. 121, No. 5, 1995, pp. 525-530. doi:10.1001/archotol.1995.01890050023005

[7] J. Stradling, G. Thomas, A Warley, et al., "Effect of Adenotonsillectomy on Nocturnal Hypoxaemia, Sleep Disturbance, and Symptoms in Snoring Children," Lancet, Vol. 335, No. 8684, 1990, pp. 249-253. doi:10.1016/0140-6736(90)90068-G

[8] C. Croft, M. Brockbank, A. Wright, et al., "Obstructive Sleep Apnoea in Children Undergoing Routine Tonsillectomy and Adenoidectomy," Clinical Otolaryngology, Vol. 15, No. 4, 1990, pp. 307-314. doi:10.1111/j.1365-2273.1990.tb00474.X

[9] C. Marcus, "Management of Obstructive Sleep Apnea in Childhood," Current Opinion in Pulmonary Medicine, Vol. 3, No. 6, 1997, pp. 464-469. doi:10.1097/00063198-199711000-00014

[10] C. L. Marcus, "Sleep Disordered Breathing in Children," American Journal of Respiratory and Critical Care Medicine, Vol. 164, No. 1, 2001, pp. 16-30.

[11] American Thoracic Society, "Standards and Indications for Cardiopulmonary Sleep Studies in Children, the Official Statement of the American Thoracic Society," American Journal of Respiratory and Critical Care Medicine, Vol. 153, 1996, pp. 866-878.

[12] R. D. Chervin, K. M. Hedger, J. E. Dillon and K. J. Pituch, "Pediatric Sleep Questionnaire (PSQ): Validity and Reliability of Scales for Sleep Disordered Breathing, Snoring, Sleepiness, and Behavioral Problems," Sleep Medicine, Vol. 1, No. 1, 2000, pp. 21-32. doi:10.1016/S1389-9457(99)00009-X

[13] C. B. Croft, M. J. Brockbank, A. Wright and A. R. Swanston, "Obstructive Sleep Apnoea in Children Undergoing Routine Tonsillectomy and Adenoidectomy,” Clinical Otolaryngology, Vol. 15, No. 4, 1990, pp. 307-314. doi:10.1111/j.1365-2273.1990.tb00474.X

[14] N. J. Ali, D. Pitson and J. R. Stardling, "Sleep Disordered Breathing: Effect of Adenotonsillectomy on Behavior and Psychological Functioning," European Journal of Pediatrics, Vol. 155, No. 1, 1996, pp. 56-62.

[15] S. Isono, A. Shimada, M. Utsugi, A. Konno and T. Nishino, "Comparison of Static Mechanical Properties of the Passive Pharynx between Normal Children and Children with Sleep-Disordered Breathing," American Journal of Respiratory and Critical Care Medicine, Vol. 157, 
No. 4, 1998, pp. 1204-1212.

[16] H. Yasan, H. Dogru, M. Tuz, O. Candir, K. Uygur and M. Yariktas, "Otitis Media with Effusion and Histopathologic Properties of Adenoid Tissue," International Journal of Pediatric Otorhinolaryngology, Vol. 67, No. 11, 2003, pp. 1179-1183. doi:10.1016/S0165-5876(03)00222-2

[17] C. Guilleminault, F. Eldridge and F. B. Simmons, "Sleep Apnoea in Eight Children,” Pediatrics, Vol. 58, 1976, pp. 23-30.

[18] C. Guilleminault, R. Korobkin and R. Winkle, "A Review of 50 Children with Obstructive Sleep Apnea Syndrome,”. Lung, Vol. 159, No. 5, 1981, pp. 275-287. doi:10.1007/BF02713925

[19] C. Guilleminault, R. Winkle, R. Korobkin and B. Sim-

\section{Appendix}

Pediatric Sleep Questionnaire items that constitute the sleep-disordered breathing scale [10]. The snoring subscale includes items A2, A3, A4, and A5. The sleepiness subscale includes items B1, B2, B4, and B6.

While sleeping, does your child ...

A2 ... snore more than half the time?

A3 ... always snore?

A4 ... snore loudly?

A5 ... have "heavy" or loud breathing?

A6 ... have trouble breathing, or struggle to breathe?

Have you ever ...

A7... seen your child stop breathing during the night?

DOES YOUR CHILD ...

A24 ... tend to breathe through the mouth during the day?

A25 ... have a dry mouth on waking up in the morning?

A32 ... occasionally wet the bed?

Does your child ... mons, "Children and Nocturnal Snoring-Evaluation of the Effects of Sleep Related Respiratory Resistive Load and Daytime Functioning," European Journal of Pediatrics, Vol. 139, No. 3, 1982, pp. 165-171. doi:10.1007/BF01377349

[20] D. J. Gottlieb, R. M. Vezina, C. Chase, et al., "Symptoms of Sleep-Disordered Breathing in 5-Year-Old Children Are Associated with Sleepiness and Problem Behaviors," Pediatrics, Vol. 112, No. 4, 2003, pp. 870-877. doi:10.1542/peds.112.4.870

[21] American Academy of Pediatrics, "Section on Pediatric Pulmonology, Subcommittee on Obstructive Sleep Apnea Syndrome. Clinical Practice Guideline: Diagnosis and Management of Childhood Obstructive Sleep Apnea Syndrome,” Pediatrics, Vol. 109, 2002, pp. 704-712. doi:10.1542/peds.109.4.704

B1 ... wake up feeling unrefreshed in the morning?

B2 ... have a problem with sleepiness during the day?

B4 ... Has a teacher or other supervisor commented that your child appears sleepy during the day?

B6 ... Is it hard to wake your child up in the morning?

B7 ... Does your child wake up with headaches in the morning?

B9 ... Did your child stop growing at a normal rate at any time since birth?

B22 ... Is your child overweight?

This child often ...

C3 ... does not seem to listen when spoken to directly.

C5 ... has difficulty organizing tasks and activities.

C8 ... is easily distracted by extraneous stimuli.

$\mathrm{C} 10$... fidgets with hands or feet or squirms in seat.

$\mathrm{C} 14$... is "on the go" or often acts as if "driven by a motor”.

C18 ... interrupts or intrudes on others (e.g., butts in to conversations or games). 